\title{
Aplicação das metodologias Desirability e Simplex para otimização das propriedades mecânicas em arames de aço temperados
}

\author{
Cristie Diego Pimenta ${ }^{a *}$, Messias Borges Silva ${ }^{a}$,Valério Antonio Pamplona Salomon ${ }^{\text {, }}$ \\ Ricardo Batista Penteado ${ }^{\text {a }}$ Fabricio Maciel Gomes ${ }^{\mathrm{a}}$ \\ a*Universidade Estadual Paulista, Guaratinguetá, SP, Brasil, pimentadiego@yahoo.com.br
}

\begin{abstract}
Resumo
O objetivo desta pesquisa é mostrar a aplicação dos métodos Desirabilitye Simplex, voltados à otimização do processo de têmpera e revenimento, em arames de aço trefilados SAE 9254. Os dados foram gerados a partir da aplicação da metodologia delineamento de experimentos, com análise em blocos, e os resultados revelaram que todas as variáveis consideradas no estudo têm influência significativa na obtenção das propriedades mecânicas investigadas. A modelagem estatística foi realizada através da aplicação do método de regressão linear múltipla, que permitiu a obtenção de modelos que representam adequadamente o processo em questão. Aos resultados das variáveis resposta limite de resistência à tração, estricção e dureza foram aplicados os métodos Desirability e Simplex e o processo foi otimizado na melhor condição de ajustes das variáveis de entrada em relação às suas especificações.
\end{abstract}

Palavras-chave

Delineamento de experimentos. Regressão linear múltipla. Função desejabilidade. Metodologia Simplex.

\section{Introdução}

Este artigo tem por finalidade mostrar o estudo da influência de fatores e a otimização de múltiplas respostas das propriedades mecânicas do processo de tratamento térmico de têmpera e revenimento em arames de aço utilizados na fabricação de molas automobilísticas. Para o levantamento dos dados e modelagem estatística do processo foram utilizadas as metodologias delineamento de experimentos e regressão linear múltipla. No caso, esses métodos foram usados para auxiliar no desenvolvimento de uma modelagem estatística que substituísse a maneira tradicional de ajustar as variáveis de entrada do processo de tratamento térmico. 0 setup desse processo, atualmente, é realizado através de ensaios mecânicos de amostras piloto que, após passarem por todas as fases de um tratamento térmico de têmpera e revenimento, são encaminhadas para análise em laboratório. Os resultados obtidos nessa etapa são usados para regular o forno de têmpera, implicando considerável tempo de análise e de espera e reduzindo, consequentemente, a produtividade do processo.
Buscou-se, através da aplicação dos métodos Desirability e Simplex, a otimização, de forma simultânea, das variáveis resposta limite de resistência à tração, dureza e estricção, que são consideradas críticas nesse processo, devido à diversidade de aplicações e às exigências de clientes. A simulação computacional dos resultados dessas propriedades mecânicas, possibilitando o seu ajuste nas melhores condições em relação às suas especificações, implicaria em grande redução de custos e ganho de produtividade devido à redução de tempo de setup dos fornos de têmpera, através da eliminação da fase de testes de amostras piloto.

\section{Revisão bibliográfica}

\subsection{Tratamento térmico e ensaios mecânicos}

A queda de temperatura durante o resfriamento, na têmpera, promove transformações estruturais 
que acarretam o surgimento de tensões internas, tornando necessário, por isso, o tratamento de revenimento. 0 processo de revenimento é realizado complementarmente à têmpera, sendo especialmente importante na fabricação de aços para molas. Ele consiste em aquecer o material temperado entre $250{ }^{\circ} \mathrm{C}$ e $650{ }^{\circ} \mathrm{C}$ por um determinado tempo, para aumentar a sua ductilidade e elasticidade (Callister Junior, 2002).

Conforme representado, na Figura 1, e segundo Mayers \& Chawla (1982), em um teste de tração, o corpo de prova (C) é fixado no cabeçote (K) de uma máquina de ensaio que aplica um esforço que tende a alongá-lo até a ruptura, sendo medidas as deformações através de um aparelho chamado extensômetro (E). 0 ensaio é realizado num corpo de prova com dimensões padronizadas, para que os resultados obtidos possam ser comparados, reproduzidos e quantificados na própria máquina. Normalmente, o ensaio ocorre até a ruptura do material (o que o classifica como destrutivo) e permite medir a resistência do material e a deformação em função da tensão aplicada. Acima de certo nível de tensão, os materiais começam a se deformar plasticamente até ocorrer a ruptura, ponto em que se obtém o limite de resistência à tração.

A máquina de ensaio universal de tração é a mais utilizada e as unidades de força mais comuns são quilograma-força por milímetro quadrado (kgf/ $\mathrm{mm}^{2}$ ) ou MegaPascal (MPa). As normas técnicas mais utilizadas para a execução de ensaios mecânicos são elaboradas pelas organizações ASTM (American Society for Testing and Materials) e ABNT (Associação Brasileira de Normas Técnicas).

Estricção é um atributo de certos materiais que quando submetidos à tensão de tração apresentam grandes transformações plásticas antes de seu rompimento. Em corpos de prova de aço, a estricção é medida pela redução de área da seção transversal que ocorre antes da ruptura. A estricção é dada pelo quociente entre a variação da área transversal do corpo de prova e o valor da área inicial da seção transversal (área inicial - área final) (Mayers \& Chawla, 1982). A estricção ou redução de área normalmente é expressa em porcentagem, mostrando quanto a área transversal da seção resistiva do corpo de prova foi reduzida após a aplicação da força $(\mathrm{F})$ no ensaio de tração, conforme mostrado na Figura 2.

Segundo Callister Junior (2002), dureza é a medida da resistência de um metal à penetração. Os métodos mais comuns de se determinar a dureza de um metal são o Brinell, o Vickers e o Rockwell. Nesta pesquisa será utilizado somente o método Brinell (HB).

A obtenção dos valores de dureza Brinell (HB), conforme mostrado na Figura 3, é calculada dividindo-se a carga aplicada pela área de penetração. 0 penetrador de diâmetro (D) é uma esfera de aço temperado para materiais de dureza média ou baixa, ou de carboneto de tungstênio para materiais de

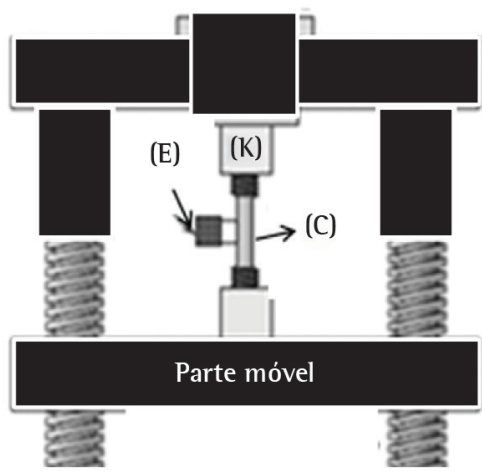

Figura 1. Representação da máquina de ensaio universal de tração. Fonte: elaboração dos autores.

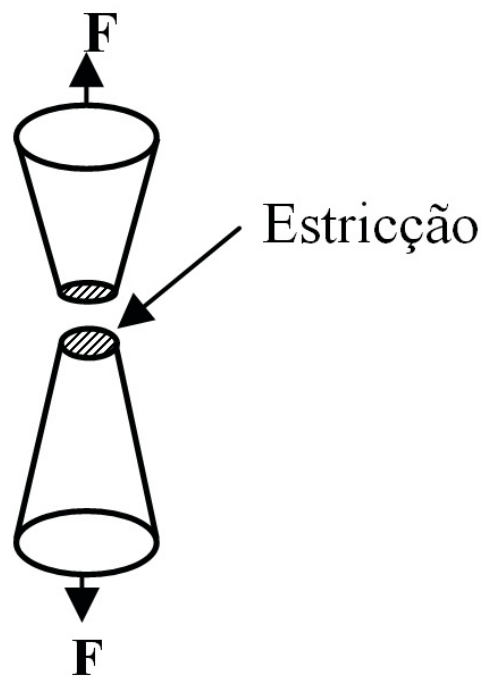

Figura 2. Redução de área após ruptura do corpo de prova submetido à tração. Fonte: elaboração dos autores.

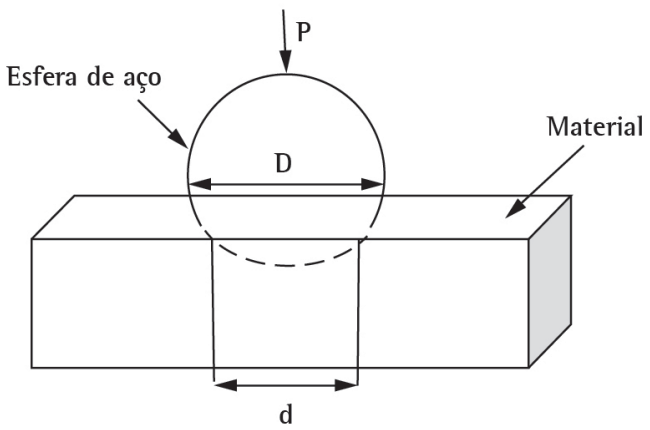

Figura 3. llustração do método de dureza Brinell (HB). Fonte: elaboração dos autores. 
elevada dureza. A máquina de ensaio possui um microscópio óptico que faz a medição do diâmetro do círculo $(d, \mathrm{em} \mathrm{mm})$ que corresponde à projeção da calota esférica impressa na amostra. A dureza Brinell (HB) será dada pela carga aplicada ( $P$, em kgf) dividida pela área de impressão, conforme mostrado na Equação 1.

$$
H B=\frac{2 P}{\pi D\left(D-\sqrt{D^{2}}-d^{2}\right)} \quad\left[\mathrm{kg} / \mathrm{mm}^{2}\right]
$$

\subsection{Métodos estatísticos}

Para Silva \& Silva (2008), o delineamento de experimentos é uma metodologia considerada poderosa para a melhoria da qualidade e produtividade em processos industriais.

Conforme Lima et al. (2011) e Granato et al. (2011), o delineamento de experimentos (DOE) é muito adequado para estudar vários fatores de processo e a complexidade de suas interações, a fim de solucionar problemas através de análises estatísticas.

A blocagem é uma técnica usada para melhorar a precisão da comparação entre fatores de interesse. Ela pode ser empregada em planejamentos fatoriais quando houver necessidade de controlar a variabilidade proveniente de fontes perturbadoras conhecidas, que podem influir nos resultados (Montgomery, 2010).

A técnica de regressão linear múltipla, quando utilizada complementarmente ao planejamento de experimentos, é muito eficiente no desenvolvimento de modelos estatísticos que quantificam a influência das variáveis de entrada do processo para predição das variáveis de saída (Benyounis \& Olabi, 2008).

Segundo Montgomery \& Runger (2003), a regressão linear múltipla é utilizada para situações que envolvem mais de um regressor e os modelos podem incluir efeitos de interação. Uma interação entre duas variáveis pode ser representada por um termo cruzado, pois se admitirmos que $x_{3}=x_{1} x_{2}$ e $\beta_{3}=\beta_{12}$, então o modelo, incluindo termos de interação, ficará como apresentado na Equação 2.

$$
Y=\beta_{0}+\beta_{1} x_{1}+\beta_{2} x_{2}+\beta_{3} x_{3}+\ldots+\varepsilon
$$

Nessa expressão, $Y$ representa a variável dependente, as variáveis independentes são representadas por $x_{1}, x_{2}, \ldots, x_{n}$ e $\varepsilon$ é o termo de erro aleatório. 0 termo linear é usado porque a equação é uma função linear dos parâmetros desconhecidos $\beta_{0}, \beta_{1}, \beta_{2}$ e $\beta_{n}$. Nesse modelo, o parâmetro $\beta_{0}$ é a interseção do plano, $\beta_{1}$, $\beta_{2}$ e $\beta_{n}$ são os coeficientes parciais de regressão.

0 método Desirability é um método utilizado para determinar as melhores condições de ajuste de processo, tornando possível a otimização simultânea de múltiplas respostas. Com isso, as melhores condições das respostas são obtidas simultaneamente, minimizando-se, maximizando-se ou buscando-se valores nominais de especificações, dependendo da situação mais conveniente para o processo (Wang $\&$ Wan, 2009).

Cada uma das respostas $\left(Y_{1}, Y_{2}, \ldots, Y_{k}\right)$ do conjunto original é transformada, tal que $d_{i}$ pertença ao intervalo $0 \leq d \leq 1$. 0 valor de $d_{i}$ aumenta quando a $i$-ésima resposta se aproxima dos limites impostos. A Equação 3 é utilizada para se encontrar o índice global $D$, a partir da combinação de cada uma das respostas transformadas através de média geométrica.

$D=\left(d_{1}\left(Y_{1}\right) \times d_{2}\left(Y_{2}\right) \ldots \times d_{k}\left(Y_{k}\right)\right)^{\frac{1}{k}}$

Como resultante da média geométrica representada pela Equação 3, o valor de $D$ avalia, de maneira geral, os níveis do conjunto combinado de respostas. É um índice também pertencente ao intervalo $[0,1]$ que será maximizado quando todas as respostas se aproximarem o máximo possível de suas especificações. Quanto mais próximo de 1 estiver $D$, mais próximas as respostas originais estarão de seus respectivos limites de especificação. 0 ponto de ótimo geral do sistema é o ponto de ótimo alcançado pela maximização da média geométrica calculada a partir das funções Desirability individuais (Paiva, 2008).

Segundo Paiva (2008), a vantagem da utilização da média geométrica é fazer com que a solução global seja alcançada de maneira balanceada, permitindo que todas as respostas atinjam os valores esperados, forçando o algoritmo a se aproximar das especificações impostas.

Segundo Derringer \& Suich (1980), o algoritmo dependerá do tipo de otimização desejada para a resposta (maximização, normalização ou minimização), dos limites desejados dentro da especificação e da importância (peso) de cada uma das respostas que identificam as principais características dos diferentes tipos de otimização, como a seguir:

- Função minimizar: 0 valor da função Desirability aumenta quando o valor da resposta original se aproxima de um valor alvo mínimo, conforme apresentado na Figura 4. Abaixo do alvo, $d=1$; acima do limite superior, $\mathrm{d}=0$.

- Função normalizar: Quando a resposta se move em direção ao alvo, o valor da função Desirability aumenta, conforme apresentado na Figura 5. Acima ou abaixo dos limites, $d=0$; no alvo, $d=1$.

- Função maximizar: 0 valor da função Desirability aumenta quando o valor da resposta aumenta. Abaixo do limite inferior, $\mathrm{d}=0$; acima do alvo, 
$\mathrm{d}=1$, conforme apresentado na Figura 6. Acima ou abaixo dos limites, $d=0$; no alvo, $d=1$.

Paiva (2008) e Wu (2005) afirmam que quando se deseja a maximização de uma resposta, a fórmula de transformação é a apresentada na Equação 4:

$$
d i= \begin{cases}0 & \hat{Y}_{i}<L S L \\ {\left[\frac{\hat{Y}_{i}-L i}{T i-L i}\right]^{R}} & L_{i} \leq \hat{Y}_{i} \leq T_{i} \\ 1 & \hat{Y}_{i}>T_{i}\end{cases}
$$

onde: $L_{i} T_{i}$ e $H_{i}$ são, respectivamente, os valores da maior, da menor e o alvo aceitáveis para a $i$-ésima resposta.

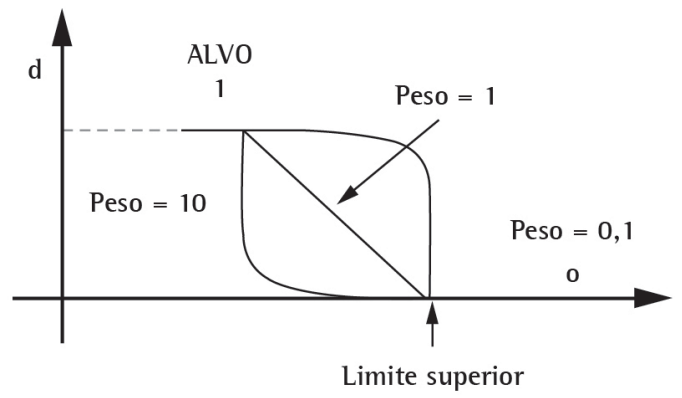

Figura 4. Função Desirability (minimizar). Fonte: elaboração dos autores.

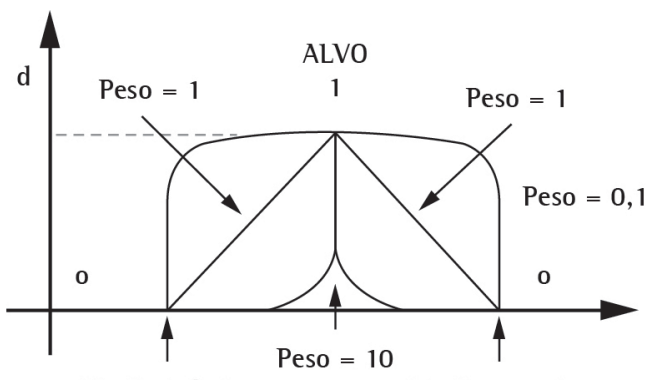

Limite inferior Limite superior

Figura 5. Função Desirability (normalizar). Fonte: elaboração dos autores.

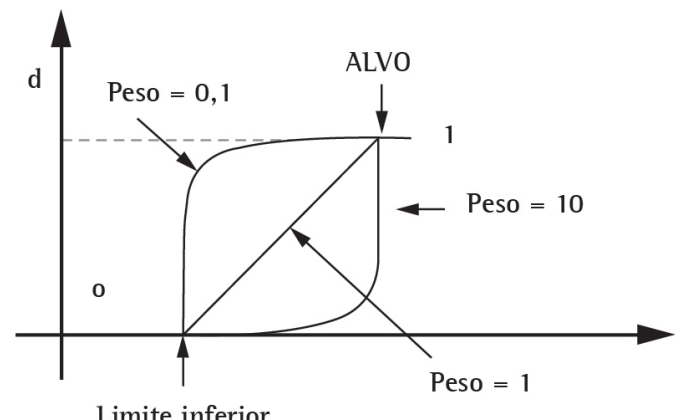

Figura 6. Função desirability (maximizar). Fonte: elaboração dos autores.
0 valor de $R$, na Equação 4, indica a preponderância do limite superior (LSL). Valores maiores que a unidade devem ser usados quando a resposta $\left(\mathrm{Y}_{\mathrm{i}}\right)$ cresce rapidamente acima de $L_{i}$ Portanto, $d_{i}$ aumenta vagarosamente, enquanto o valor da resposta vai sendo maximizado. Logo, para se maximizar $D$, a $i$-ésima resposta deve ser bem maior que $L_{i}$ Pode-se escolher $R<1$ quando encontrar valores para a resposta abaixo dos limites fixados não for crítico.

Em casos em que o objetivo é atingir um valor alvo, a formulação de transformação deixa de ser unilateral e passa a ser bilateral. A formulação bilateral, representada pela Equação 5, ocorre quando a resposta de interesse possui duas restrições: uma de máximo e outra de mínimo.

$$
d_{i}= \begin{cases}0 & \hat{Y}_{i}<L_{i} \text { ou } \hat{Y}_{i}<H_{i} \\ {\left[\frac{H_{i}-\hat{Y}_{i}}{H_{i}-T i}\right]^{R}} & T_{i} \leq \hat{Y}_{i} \leq H_{i} \\ {\left[\frac{\hat{Y}_{i}-L i}{T i-L i}\right]^{R}} & L_{i} \leq \hat{Y}_{i} \leq T_{i}\end{cases}
$$

0 método Simplex (por programação linear) tem por objetivo encontrar a melhor solução para problemas que tenham seus modelos representados por expressões lineares. Consiste na maximização ou minimização de uma função objetivo, respeitando um sistema de igualdades ou desigualdades, que recebem o nome de restrições do modelo. Essas restrições determinam uma região à qual se dá o nome de conjunto viável ou solução ótima (Marins, 2011).

Segundo Marins (2011), o método Simplex é um método de programação linear que fornece a solução de qualquer modelo linear, podendo ser utilizado para a sua aplicação o "solver" do Excel ${ }^{\circledR}$, que atua com planilhas eletrônicas e indica se o modelo tem solução ilimitada, se não tem solução ou se possui infinitas soluções.

\section{Materiais e métodos}

\subsection{Material, seleção dos fatores e organização experimental}

0 material utilizado foi o arame de aço SAE 9254 com bitolas de diâmetro 2,00 $\mathrm{mm}$ e $6,50 \mathrm{~mm}$. Os fatores investigados, nesta pesquisa, foram:

- Velocidade de passagem do arame dentro do forno (em m/s);

- Concentração do polímero, meio de têmpera (em \%);

- Temperatura do chumbo no revenimento (em ${ }^{\circ} \mathrm{C}$ ). 
0 diâmetro do arame de aço também foi considerado um fator importante, pois existia a hipótese de que sua massa pudesse influenciar nos resultados das propriedades mecânicas investigadas. Nesta pesquisa, porém, foi utilizada a metodologia de análise em blocos, ou seja, para o bloco 1 foram alocados os experimentos relacionados somente ao diâmetro de 2,00 $\mathrm{mm}$ e, para o bloco 2, os experimentos relacionados ao diâmetro de $6,50 \mathrm{~mm}$, conforme mostrado na Tabela 1.

Os fatores velocidade, temperatura do chumbo e concentração do polímero foram experimentados através dos planejamentos fatoriais utilizando-se a matriz $2^{3}$.

Para a realização do planejamento de experimentos foram utilizadas variáveis reduzidas ( $\beta$ ) ao invés de variáveis físicas (ajustes reais) dos fatores investigados, de forma a se preservarem os dados confidenciais da empresa financiadora da pesquisa. A redução das variáveis foi calculada segundo Montgomery \& Runger (2003), utilizando-se o valor físico $(\alpha)$ que se quer testar subtraído da média $(\mu)$ entre os valores mínimo e máximo dos ajustes dos fatores. 0 resultado foi dividido pela metade da amplitude (R) entre os valores mínimos e máximos dos ajustes dos fatores. Dessa forma, a dimensionalidade das variáveis reduzidas ficou restrita ao intervalo [-1 a 1], conforme Equação 6 e Tabela 2.

$\beta=\frac{\alpha-\mu}{\frac{R}{2}}$

\section{Resultados e discussão}

\subsection{Experimentação e análise estatística}

$\mathrm{Na}$ experimentação foram realizadas inicialmente todas as réplicas relacionadas ao bloco $1 \mathrm{e}$, em seguida, as correspondentes ao bloco 2 . Seis réplicas foram utilizadas para cada condição experimental. As replicações foram aleatorizadas e sequenciadas, utilizando uma numeração de 1 a 8 , correspondente à ordem de realização de cada experimento, para cada bloco, individualmente. Essa sequência de experimentação é apresentada entre parênteses e em formato subscrito ao lado dos valores das propriedades mecânicas obtidos, conforme mostrado nas Tabelas 3, 4 e 5. É possível observar, ainda, que para cada condição experimental determinaram-se os valores das três propriedades mecânicas estudadas, correspondentes a cada réplica realizada.

A significância dos fatores foi testada a um nível de $95 \%$ de confiança $(p<0,05)$. Essa análise foi realizada separadamente para que pudesse ser
Tabela 1. Matriz fatorial $2^{3}$.

\begin{tabular}{cccc}
\hline Experimentos & Velocidade & $\begin{array}{c}\text { Temperatura } \\
\text { chumbo }\end{array}$ & \% polimero \\
\hline 1 & - & - & - \\
2 & + & - & - \\
3 & - & + & - \\
4 & + & + & - \\
5 & - & - & + \\
6 & + & - & + \\
7 & - & + & + \\
8 & + & + & + \\
\hline
\end{tabular}

Tabela 2. Transformação de variáveis físicas para variáveis reduzidas.

\begin{tabular}{ccc}
\hline Variáveis de entrada & Unidades físicas & Variáveis reduzidas \\
\hline Velocidade $(\mathrm{m} / \mathrm{s})$ & Mínimo/Máximo & $-1 / 1$ \\
$\begin{array}{c}\text { Temperatura do } \\
\text { chumbo }\left({ }^{\circ} \mathrm{C}\right)\end{array}$ & Mínimo/Máximo & $-1 / 1$ \\
$\begin{array}{c}\text { Concentração do } \\
\text { polímero }(\%)\end{array}$ & Mínimo/Máximo & $-1 / 1$ \\
\hline
\end{tabular}

verificada a significância dos fatores para cada uma das respostas das propriedades mecânicas estudadas, conforme mostradas nas Tabelas 6, 7 e 8.

Através do teste de significância realizado para a propriedade mecânica limite de resistência à tração (mostrado na Tabela 6), foi constatado que os fatores significativos (em que $p<0,05)$ são: diâmetro do arame (representado pela letra D e testado através de blocos), velocidade (representada pela letra $A$ ), temperatura do chumbo (representado pela letra B), concentração do polímero (representada pela letra C), interações de segunda ordem entre velocidade e concentração do polímero, temperatura e concentração do polímero e uma interação de terceira ordem entre velocidade, temperatura do chumbo e concentração do polímero.

Analisando-se o teste de significância para a propriedade mecânica estricção (mostrado na Tabela 7), é possivel constatar que os fatores influentes (em que $p<0,05)$ são: diâmetro do arame (testado através de blocos), velocidade, temperatura do chumbo, concentração do polímero, interações de segunda ordem entre velocidade e temperatura do chumbo, velocidade e concentração do polímero, temperatura e concentração do polímero e uma interação de terceira ordem entre velocidade, temperatura do chumbo e concentração do polímero.

Analisando-se o teste de significância para a propriedade mecânica dureza (mostrada na Tabela 8), é possível afirmar que os fatores influentes (no qual $\mathrm{p}<0,05)$ são: diâmetro do arame (testado através de blocos), velocidade, temperatura do chumbo, concentração do polímero, interações de segunda ordem entre velocidade e concentração do polímero, 
Pimenta, C. D. et al.

Aplicação das metodologias Desirability ... arames de aço temperados. Production, v. 25, n. 3, p. 598-610, jul./set. 2015

Tabela 3. Resultados de tração (MPa).

\begin{tabular}{|c|c|c|c|c|c|c|}
\hline Experimentos & Réplica 1 & Réplica 2 & Réplica 3 & Réplica 4 & Réplica 5 & Réplica 6 \\
\hline 1/Bloco 1 & 2149 (1) & 2148 (1) & $2146_{(2)}$ & $2161_{(8)}$ & 2167 (1) & $2160_{(6)}$ \\
\hline 2/Bloco 1 & 2157 & $2155_{(7)}$ & 2157 & $2151_{(7)}$ & $2157_{(4)}$ & $2157_{(2)}$ \\
\hline 3/Bloco 1 & $1924_{(3)}$ & 1922 & 1920 & $1921_{(5)}$ & $1920_{(6)}$ & $1918_{(4)}$ \\
\hline 4/Bloco 1 & 1924 & 1924 & 1922 & 1943 (6) & $1945_{(8)}$ & 1945 \\
\hline 5/Bloco 1 & $2108_{(6)}$ & $2106_{(5)}$ & $21088_{(7)}$ & $2104_{(2)}$ & $2102_{(7)}$ & $2109_{(8)}$ \\
\hline 6/Bloco 1 & $2136_{(5)}$ & 2127 & 2127 & $2136_{(3)}$ & $2134_{(3)}$ & $2127_{(3)}$ \\
\hline 7/Bloco 1 & 1927 & 1926 & $1944_{(5)}$ & 1935 (4) & $1946_{(2)}$ & 1947 \\
\hline 8/Bloco 1 & 1946 & 1946 (6) & 1946 (6) & 1953 & 1951 & 1946 \\
\hline 1/Bloco 2 & 1968 & $1974_{(1)}$ & 1962 & $1971_{(4)}$ & $1971_{(8)}$ & $1974_{(5)}$ \\
\hline 2/Bloco 2 & $1980_{(7)}$ & $1976_{(4)}$ & $1988_{(6)}$ & $1978_{(2)}$ & $1980_{(3)}$ & $1988_{(2)}$ \\
\hline 3/Bloco 2 & 1771 & $1764_{(3)}$ & 1763 & 1773 & 1771 & 1764 \\
\hline 4/Bloco 2 & $1796_{(8)}$ & $1784_{(2)}$ & $17977_{(8)}$ & $1781_{(3)}$ & $1796_{(2)}$ & $1784_{(3)}$ \\
\hline 5/Bloco 2 & 1949 & 1963 & 1947 (1) & 1951 & 1949 & $1947_{(6)}$ \\
\hline 6/Bloco 2 & $1992_{(4)}$ & $1980_{(5)}$ & $1976_{(4)}$ & $1994_{(8)}$ & $1980_{(7)}$ & $1992_{(7)}$ \\
\hline 7/Bloco 2 & $1760_{(2)}$ & $1768_{(7)}$ & $1766_{(5)}$ & 1763 (7) & $1766_{(6)}$ & $1763{ }_{(8)}$ \\
\hline 8/Bloco 2 & 1787 & 1793 & $1785_{(2)}$ & $1784_{(6)}$ & 1784 & 1785 \\
\hline
\end{tabular}

Tabela 4. Resultados de estricção em porcentagem (\%).

\begin{tabular}{|c|c|c|c|c|c|c|}
\hline Experimentos & Réplica 1 & Réplica 2 & Réplica 3 & Réplica 4 & Réplica 5 & Réplica 6 \\
\hline 1/Bloco 1 & 50 & $51_{(1)}$ & $51_{(2)}$ & $50_{(8)}$ & 50 (1) & $50_{(6)}$ \\
\hline 2/Bloco 1 & 50 & $50_{(7)}$ & 50 & 50 & 50 & $50_{(2)}$ \\
\hline 3/Bloco 1 & 58 & 58 & 58 & $58_{(5)}$ & 58 & 58 \\
\hline 4/Bloco 1 & $58^{(2)}$ & 58 & $58_{(8)}$ & $56_{(6)}$ & $56_{(8)}$ & $56_{(5)}$ \\
\hline 5/Bloco 1 & 53 (6) & 53 & 53 (7) & 53 & 53 & $53(8)$ \\
\hline 6/Bloco 1 & 51 & $52_{(4)}$ & $52_{(4)}$ & $51_{(3)}$ & $51_{(3)}$ & 52 (3) \\
\hline 7/Bloco 1 & 58 & $58_{(2)}$ & 56 & $58{ }_{(4)}$ & $56_{(2)}$ & $56_{(7)}$ \\
\hline 8/Bloco 1 & $56_{(8)}$ & $56_{(6)}$ & $56_{(6)}$ & $55_{(1)}$ & $56_{(5)}$ & 56 \\
\hline 1/Bloco 2 & 42 & 41 & 42 & $42_{(4)}$ & 42 & $41_{(5)}$ \\
\hline 2/Bloco 2 & $41_{(7)}$ & $41_{(4)}$ & $40_{(6)}$ & $41_{(2)}$ & $41_{(3)}$ & $40_{(2)}$ \\
\hline 3/Bloco 2 & $47_{(3)}$ & $46_{(3)}$ & $46_{(7)}$ & $47_{(5)}$ & $47_{(5)}$ & $46_{(4)}$ \\
\hline 4/Bloco 2 & $44_{(8)}$ & $45_{(2)}$ & $44_{(8)}$ & $45_{(3)}$ & $44_{(2)}$ & $45_{(3)}$ \\
\hline 5/Bloco 2 & $56_{(5)}$ & 42 (6) & 56 & $566_{(1)}$ & $56_{(4)}$ & 56 (6) \\
\hline 6/Bloco 2 & $40_{(4)}$ & $41_{(5)}$ & $41_{(4)}$ & $40_{(8)}$ & $41_{(7)}$ & 40 \\
\hline 7/Bloco 2 & $46_{(2)}$ & 47 & 47 & $46_{(7)}$ & 47 (6) & $46_{(8)}$ \\
\hline 8/Bloco 2 & $44_{(6)}$ & $44_{(8)}$ & $45_{(2)}$ & $45_{(6)}$ & 45 & 45 \\
\hline
\end{tabular}

Tabela 5. Resultados de dureza (Dureza Brinell).

\begin{tabular}{|c|c|c|c|c|c|c|}
\hline Experimentos & Réplica 1 & Réplica 2 & Réplica 3 & Réplica 4 & Réplica 5 & Réplica 6 \\
\hline 1/Bloco 1 & $608_{(1)}$ & $606_{(1)}$ & $606_{(2)}$ & $611_{(8)}$ & $611_{(1)}$ & $611_{(6)}$ \\
\hline 2/Bloco 1 & 608 & $608_{(7)}$ & 608 & $608_{(7)}$ & $6088_{(4)}$ & $608_{(2)}$ \\
\hline 3/Bloco 1 & $544_{(3)}$ & $542_{(3)}$ & 542 (1) & $542_{(5)}$ & 542 (6) & $542_{(4)}$ \\
\hline 4/Bloco 1 & $544_{(2)}$ & $544_{(8)}$ & $542_{(8)}$ & 550 & 550 & 550 \\
\hline 5/Bloco 1 & $594_{(6)}$ & $594_{(5)}$ & $594_{(7)}$ & $594_{(2)}$ & $594_{(7)}$ & $594_{(8)}$ \\
\hline 6/Bloco 1 & 603 & $600_{(4)}$ & $600_{(4)}$ & 603 & 603 & $600_{(3)}$ \\
\hline 7/Bloco 1 & $544_{(7)}$ & $544_{(2)}$ & $550_{(5)}$ & $547_{(4)}$ & $550_{(2)}$ & $550_{(7)}$ \\
\hline 8/Bloco 1 & $550_{(8)}$ & $550_{(6)}$ & $550_{(6)}$ & 553 (1) & $550_{(5)}$ & 550 (1) \\
\hline 1/Bloco 2 & 556 & 558 & $556_{(3)}$ & $556_{(4)}$ & $556_{(8)}$ & 558 \\
\hline 2/Bloco 2 & $558_{(7)}$ & $5588_{(4)}$ & $561_{(6)}$ & $558_{(2)}$ & $558_{(3)}$ & $561_{(2)}$ \\
\hline 3/Bloco 2 & $500_{(3)}$ & 497 & 497 & $500_{(5)}$ & $500_{(5)}$ & 497 \\
\hline 4/Bloco 2 & 508 (8) & 503 (2) & 508 & 503 & $5088_{(2)}$ & 503 \\
\hline 5/Bloco 2 & 550 & $556_{(6)}$ & 550 & 550 (1) & $550_{(4)}$ & $550_{(6)}$ \\
\hline 6/Bloco 2 & $564_{(4)}$ & 558 & 558 & 564 & 558 & 564 \\
\hline 7/Bloco 2 & 497 & $500_{(7)}$ & 500 & 497 & 500 & 497 \\
\hline 8/Bloco 2 & $506_{(6)}$ & $506_{(8)}$ & $503_{(2)}$ & 503 (6) & 503 (1) & 503 (1) \\
\hline
\end{tabular}


Tabela 6. Teste de significância para limite de resistência (em MPa).

\begin{tabular}{crrrc}
\hline Termos & Efeito & Coeficiente & \multicolumn{1}{c}{$\mathrm{T}$} & $\mathrm{p}$ \\
\hline Constante & & 1955,29 & 1782,89 & 0,000 \\
(D) & 165,62 & 82,81 & 80,09 & 0,000 \\
(A) & 17,42 & 8,71 & 7,94 & 0,000 \\
$(\mathrm{~B})$ & $-198,54$ & $-99,27$ & $-90,52$ & 0,000 \\
(C) & $-8,04$ & $-4,02$ & $-3,67$ & 0,000 \\
$(\mathrm{~A})(\mathrm{B})$ & $-0,54$ & $-0,27$ & $-0,25$ & 0,805 \\
$(\mathrm{~A})(\mathrm{C})$ & 5,62 & 2,81 & 2,56 & 0,012 \\
$(\mathrm{~B})(\mathrm{C})$ & 14,08 & 7,04 & 6,42 & 0,000 \\
$(\mathrm{~A})(\mathrm{B})(\mathrm{C})$ & $-6,25$ & $-3,13$ & $-2,85$ & 0,005 \\
\hline
\end{tabular}

Tabela 7. Teste de significância para estricção (em porcentagem).

\begin{tabular}{crrrc}
\hline Termos & Efeito & Coeficiente & \multicolumn{1}{c}{$\mathrm{T}$} & $\mathrm{p}$ \\
\hline Constante & & 49,458 & 201,94 & 0,000 \\
(D) & 9,426 & 4,713 & 201,94 & 0,000 \\
(A) & $-2,750$ & $-1,375$ & $-5,61$ & 0,000 \\
(B) & 3,583 & 1,792 & 7,32 & 0,000 \\
(C) & 1,750 & 0,875 & 3,57 & 0,001 \\
(A)(B) & 1,250 & 0,625 & 2,55 & 0,012 \\
(A)(C) & 1,667 & $-0,833$ & $-3,40$ & 0,001 \\
(B)(C) & $-2,250$ & $-1,125$ & $-4,59$ & 0,000 \\
(A)(B)(C) & 1,667 & 0,833 & 3,40 & 0,001 \\
\hline
\end{tabular}

Tabela 8. Teste de significância para dureza (em HB).

\begin{tabular}{crrrc}
\hline Termos & Efeito & Coeficiente & \multicolumn{1}{c}{ T } & $p$ \\
\hline Constante & & 552,09 & 1650,05 & 0,000 \\
(D) & 46,86 & 23,43 & 74,26 & 0,000 \\
(A) & 4,85 & 2,43 & 7,25 & 0,000 \\
(B) & $-55,81$ & $-27,91$ & $-83,40$ & 0,000 \\
(C) & $-2,19$ & $-1,09$ & $-3,27$ & 0,001 \\
(A)(B) & 0,10 & 0,05 & 0,16 & 0,877 \\
(A)(C) & 1,65 & 0,82 & 2,46 & 0,016 \\
(B)(C) & 4,06 & 2,03 & 6,07 & 0,000 \\
(A)(B)(C) & $-2,35$ & $-1,18$ & $-3,52$ & 0,001 \\
\hline
\end{tabular}

temperatura e concentração do polímero e uma interação de terceira ordem entre velocidade, temperatura do chumbo e concentração do polímero.

\subsection{Modelagem estatística para as múltiplas respostas}

Utilizando-se os coeficientes calculados através do teste de significância, foi possível a construção de modelos estatísticos que representam a relação entre as variáveis de entrada do processo (fatores) e as variáveis de saída (propriedades mecânicas). Tais modelos estatísticos estão definidos nas Equações 7, 8 e 9.

$\mathrm{LR}=1955,29+82,81(\mathrm{D})+8,71(\mathrm{~A})$

$-99,27(\mathrm{~B})-4,02(\mathrm{C})+2,81(\mathrm{~A})(\mathrm{C})$

$$
\begin{aligned}
& E=49,458+4,713(D)-1,375(A) \\
& +1,792(B)+0,875(C)+0,625(A)(B) \\
& -0,833(A)(C)-1,125(B)(C)+0,833(A)(B)(C) \\
& \\
& D=552,09+23,43(D)+2,43(A) \\
& -27,91(B)-1,09(C)+0,82(A)(C) \\
& +2,03(B)(C)-1,18(A)(B)(C)
\end{aligned}
$$

onde: LR corresponde à variável resposta limite de resistência à tração, E corresponde à variável resposta estricção, e D corresponde à variável resposta dureza.

\subsection{Aplicação da função Desirability para otimização}

Para a otimização do processo através da utilização da função Desirability, primeiramente foi necessária a definição das especificações requeridas para as propriedades mecânicas estudadas. Para isso foram analisados os blocos separadamente, ou seja, otimizaram-se as variáveis resposta, primeiramente, para o arame com diâmetro de 2,00 $\mathrm{mm}$ e, posteriormente, realizou-se o mesmo procedimento para de o diâmetro $6,5 \mathrm{~mm}$.

As especificações (mínima, nominal e máxima) referentes ao diâmetro 2,00 mm são apresentadas na Tabela 9. Nesse caso, buscam-se valores nominais (alvo) para as propriedades mecânicas limite de resistência à tração e dureza e, para a propriedade mecânica estricção, busca-se a maximização, pois quanto maior o valor, melhor será para o produto em questão.

0 composto Desirability (D) é o índice global, calculado a partir da combinação de cada uma das variáveis resposta transformadas através de uma média geométrica; esse índice é responsável por mostrar a melhor condição para otimização de todas as variáveis resposta ao mesmo tempo. Para a obtenção do maior valor possível para $\mathrm{D}$, que se reflete na melhor condição das variáveis resposta em relação ao atendimento das suas especificações (apresentada na Figura 7), os melhores ajustes, utilizando-se variáveis reduzidas [-1 a 1] dos fatores são:

- Velocidade, ajustada em - 1,0;

- Temperatura do chumbo, ajustada em - 0,0909;

- Concentração do polímero, ajustada em 1,0.

Analisando-se a Figura 7 é possível constatar que o valor de $D$, pertencente ao intervalo [0-1], é maximizado quando todas as respostas se aproximam de suas especificações, pois quanto mais próximo de 1 estiver $D$, mais próximas as respostas originais estarão dos seus respectivos limites de especificação. 0 ponto de ótimo geral do sistema é o ponto de ótimo 
Tabela 9. Especificações para o diâmetro 2,00 mm.

\begin{tabular}{|c|c|c|c|c|c|c|c|c|}
\hline \multicolumn{3}{|c|}{ Limite de resistência à tração (MPa) } & \multicolumn{3}{|c|}{ Estricção (\%) } & \multicolumn{3}{|c|}{ Dureza (HB) } \\
\hline Mínima & $\begin{array}{c}\text { Nominal } \\
\text { (alvo) }\end{array}$ & Máxima & Mínima & Nominal & $\begin{array}{l}\text { Máxima } \\
\text { (alvo) }\end{array}$ & Mínima & $\begin{array}{c}\text { Nominal } \\
\text { (alvo) }\end{array}$ & Máxima \\
\hline 1930 & 2040 & 2150 & 40 & 45 & 50 & 545 & 572 & 60 \\
\hline
\end{tabular}

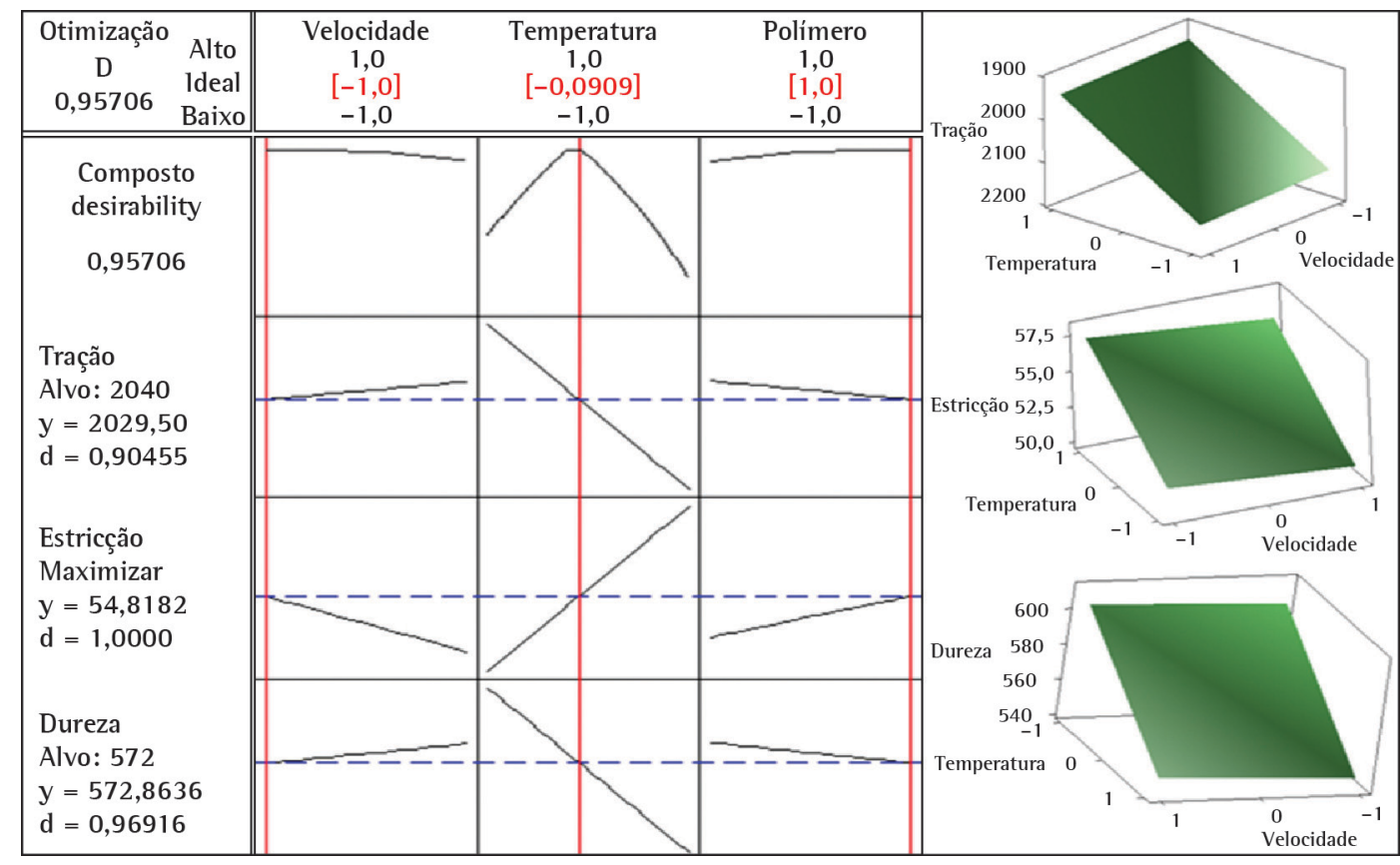

Figura 7. Função Desirability e superfícies das resposta tração, estricção e dureza (diâmetro 2,00 mm).

alcançado pela maximização da média geométrica, calculada a partir das funções Desirability individuais (d) que, nesse caso, são os valores para cada uma das variáveis resposta dados a seguir:

- Para a variável resposta limite de resistência à tração, temos $\mathrm{d}=0,90455$;

- Para a variável resposta estricção, temos $d=1,0$;

- Para a variável resposta dureza, temos $\mathrm{d}=0,96916$.

Os valores obtidos para o composto Desirability (D) e Desirability individuais (d) demonstram que o processo foi bem otimizado, pois esses índices se encontram muito próximos à condição ótima $(1,0)$. Dessa forma foi possível constatar que os valores obtidos para essa condição otimizada atendem às especificações requeridas e são:

- Para limite de resistência à tração,y = 2029,5 MPa;

- Para estricção, y = 54,8182\%;

- Para dureza, y = 572,8636 HB.

Analisando-se a Figura 7 foi possível constatar que o fator velocidade, ao ser aumentado, provoca também o aumento dos valores das variáveis resposta limite de resistência à tração (MPa) e dureza (HB). Também, o aumento da velocidade repercute na redução da variável resposta estricção (\%) e redução do composto Desirability (D).

Em relação ao fator temperatura do chumbo, com o aumento da temperatura percebe-se a redução dos valores das variáveis resposta limite de resistência à tração (MPa), dureza (HB) e do composto Desirability (D). Por outro lado, aumenta o valor da estricção (\%).

Observando-se o aumento do fator concentração do polímero é possível perceber que haverá queda dos valores das variáveis resposta limite de resistência à tração (MPa) e dureza (HB), aumentando a estricção (\%) e o composto Desirability (D).

Na Tabela 10, são mostradas as especificações (mínima, nominal e máxima) referentes ao diâmetro de $6,50 \mathrm{~mm}$. Também buscam-se valores nominais (alvo) para as propriedades mecânicas limite de resistência à tração e dureza e a maximização para a propriedade mecânica estricção.

Conforme mostrado na Figura 8, para a obtenção do maior valor possível para o composto Desirability (D), os melhores ajustes dos fatores são:

- Velocidade, ajustada em - 1,0;

- Temperatura do chumbo, ajustada em - 0,1919; 
Tabela 10. Especificações para o diâmetro $6,50 \mathrm{~mm}$.

\begin{tabular}{|c|c|c|c|c|c|c|c|c|}
\hline \multicolumn{3}{|c|}{ Limite de resistência à tração (MPa) } & \multicolumn{3}{|c|}{ Estricção (\%) } & \multicolumn{3}{|c|}{ Dureza (HB) } \\
\hline Mínima & $\begin{array}{c}\text { Nominal } \\
\text { (alvo) }\end{array}$ & Máxima & Mínima & Nominal & $\begin{array}{l}\text { Máxima } \\
\text { (alvo) }\end{array}$ & Mínima & $\begin{array}{c}\text { Nominal } \\
\text { (alvo) }\end{array}$ & Máxima \\
\hline 1770 & 1875 & 1980 & 40 & 48 & 56 & 500 & 530 & 560 \\
\hline
\end{tabular}

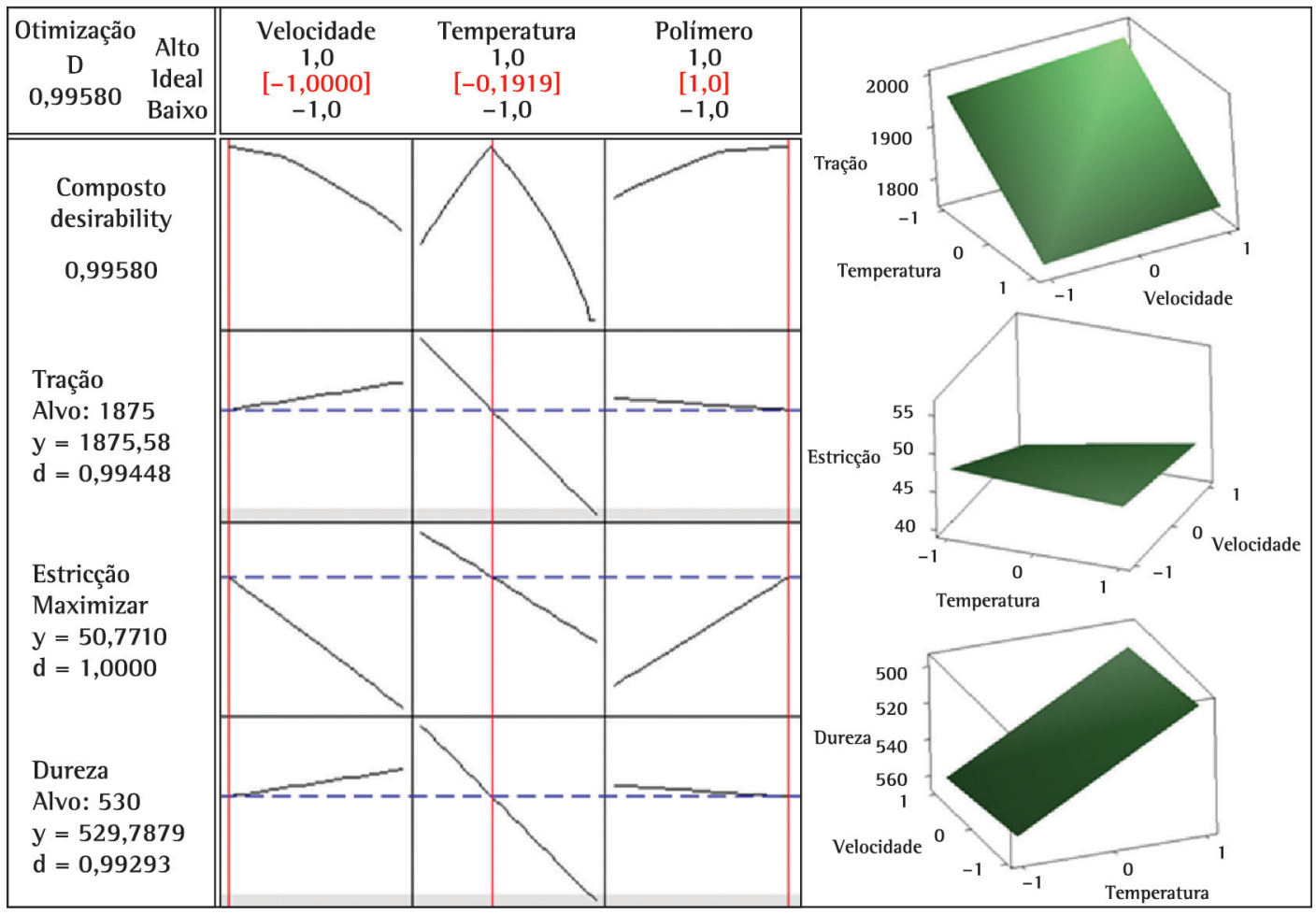

Figura 8. Função Desirability e superfícies das resposta tração, estricção e dureza (diâmetro 6,50 mm).

- Concentração do polímero, ajustada em 1,0.

Através da análise da Figura 8 é possível constatar que:

- Para a variável resposta limite de resistência à tração temos d =0,99448;

- Para a variável resposta estricção temos $d=1,0$;

- Para a variável resposta dureza, temos $d=0,99293$.

Também é possível observar que os valores obtidos para essa condição otimizada atendem às especificações requeridas e são:

- Para limite de resistência à tração, $\mathrm{y}=1875,5791 \mathrm{MPa}$;

- Para estricção, y = 50,7710 \%;

- Para dureza, y = 529,7879 HB.

E que, em relação ao fator velocidade, ao se aumentar a velocidade se obtém aumento dos valores das variáveis resposta limite de resistência à tração (MPa) e dureza (HB). Também, com o aumento do fator velocidade, percebe-se a redução da variável resposta estricção (\%) e a redução do composto Desirability (D).

Em relação ao fator temperatura do chumbo, o aumento faz com que haja a redução de todas as variáveis resposta, inclusive do composto Desirability (D).

Observando-se o fator concentração do polímero, constata-se que o aumento provocará queda das variáveis resposta limite de resistência à tração e dureza, aumentando a estricção e o composto Desirability (D).

A linha vermelha (vertical) na Figura 8 pode ser interpretada da seguinte forma: caso seja movimentada, irá alterar os valores das respostas e isso afetará diretamente os valores do composto Desirability (D) e dos Desirability individuais (d). Por exemplo, ao se movimentar a linha vermelha no espaço referente ao fator temperatura do chumbo para o lado direito, proporcionar-se-á queda no composto Desirability (D) e de todas as variáveis resposta (mostrado na Figura 8). É possivel perceber a queda, no composto 
Desirability (D), observando-se a inclinação da reta contida no local indicado anteriormente. Essa queda em $\mathrm{D}$ representaria a redução da otimização das múltiplas respostas e, consequentemente, a não utilização das respostas em suas melhores condições de ajuste dos fatores.

\subsection{Aplicação do método Simplex para otimização}

\subsubsection{Aplicação para o diâmetro de $2,00 \mathrm{~mm}$}

Além da aplicação do método Desirability, também buscou-se a otimização do processo através da aplicação do método Simplex (por programação linear), por meio da ferramenta "solver" do software Excel ${ }^{\circledR}$, versão 2010.

Para a aplicação desse método foi necessária a definição de:

- Especificações: Para otimização do diâmetro de 2, 00mm (Tabela 9); onde se buscam para a variável resposta limite de resistência à tração valores entre $1930 \mathrm{MPa}$ a $2150 \mathrm{MPa}$, para estricção, valores entre $40 \%$ a $55 \%$ e, para dureza, valores entre $545 \mathrm{HB}$ a 600 HB. Essas especificações serão inseridas em forma

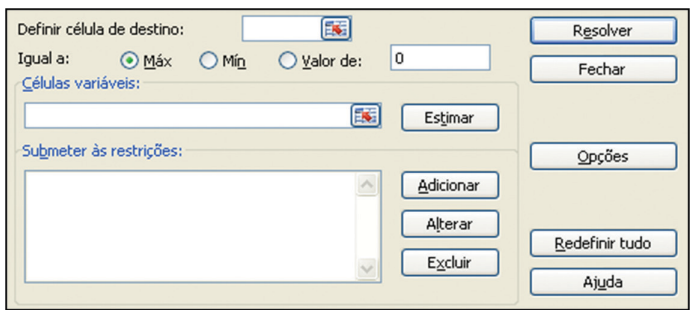

Figura 9. Tela de utilização da ferramenta "solver" (Excel ${ }^{\circledR}$ versão 2010). de restrições, no campo "submeter às restrições" e em seguida "adicionar", conforme mostrado nas Figuras 9 e 10.

- Variáveis de decisão: Nesse caso, referem-se aos valores de ajustes dos fatores A, B e C, para que seja proporcionada a melhor condição de atendimento às especificações das múltiplas respostas das propriedades mecânicas. As variáveis de decisão serão inseridas no campo "células variáveis", conforme utilização da ferramenta "solver", apresentada na Figura 9.

- Função objetivo: É a média da distância que foi alcançada na predição de cada variável resposta em comparação à sua especificação nominal, em porcentagem (\%), ou seja, para cada resposta predita pelo modelo, subtrai-se o valor "alvo", então se obtém uma unidade de erro entre a predição e a especificação nominal. Nesse caso, a função objetivo será a minimização da média desses "erros", buscando-se assim uma condição que reduza ao máximo os valores das distâncias simultaneamente, proporcionando o melhor atendimento das especificações nominais através dos melhores ajustes dos fatores. A função objetivo será inserida no campo "definir célula de destino", utilizando-se a ferramenta "solver" conforme mostrado na Figura 9.

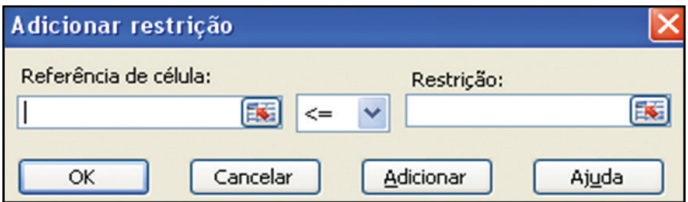

Figura 10. Tela "adicionar restrição" para inserção das restrições (Excel ${ }^{\circledR}$, versão 2010).

Tabela 11. Simulação usando o modelo matemático (diâmetro 2,00mm).

\begin{tabular}{|c|c|c|c|c|c|c|c|c|c|c|c|c|}
\hline \multirow[b]{2}{*}{ Resposta } & \multirow{2}{*}{$\begin{array}{l}\text { Média } \\
\text { (resposta) }\end{array}$} & \multicolumn{11}{|c|}{ Fatores } \\
\hline & & D & A & B & C & $A B$ & $\mathrm{AC}$ & $\mathrm{BC}$ & $\mathrm{ABC}$ & $\begin{array}{c}\text { Respostas } \\
\text { obtidas }\end{array}$ & Erro & $\begin{array}{c}\text { Erro } \\
\text { médio }\end{array}$ \\
\hline Tração & 1955,29 & 82,81 & 8,71 & $-99,27$ & $-5,3$ & - & 2,81 & 7,04 & -13 & 2040 & 1,57 & \\
\hline Estricção & 49,458 & 4,113 & $-1,38$ & 1,792 & 0,88 & 0,63 & $-0,83$ & $-1,13$ & 0,83 & 53,5 & 15,9 & $4,28 \%$ \\
\hline Dureza & 552,09 & 23,43 & 2,43 & $-27,91$ & $-1,09$ & - & 0,82 & 2,03 & $-1,18$ & 576,1 & 0,71 & \\
\hline \multicolumn{2}{|c|}{ Melhor ajuste } & -1 & 1 & 0,042 & -1 & - & - & - & - & - & - & - \\
\hline
\end{tabular}

Tabela 12. Simulação usando o modelo matemático (diâmetro 6,50 mm).

\begin{tabular}{|c|c|c|c|c|c|c|c|c|c|c|c|c|}
\hline \multirow[b]{2}{*}{ Resposta } & \multirow{2}{*}{$\begin{array}{c}\text { Média } \\
\text { (resposta) }\end{array}$} & \multicolumn{11}{|c|}{ Fatores } \\
\hline & & $\mathrm{D}$ & A & B & C & $\mathrm{AB}$ & $A C$ & $\mathrm{BC}$ & $A B C$ & $\begin{array}{l}\text { Respostas } \\
\text { obtidas }\end{array}$ & Erro & $\begin{array}{l}\text { Erro } \\
\text { médio }\end{array}$ \\
\hline Tração & 1955,29 & 82,81 & 8,71 & $-99,27$ & $-5,3$ & - & 2,81 & 7,04 & -13 & 2040 & 1,57 & \\
\hline Estricção & 49,458 & 4,113 & $-1,38$ & 1,792 & 0,88 & 0,63 & $-0,83$ & $-1,13$ & 0,83 & 53,5 & 15,9 & $4,28 \%$ \\
\hline Dureza & 552,09 & 23,43 & 2,43 & $-27,91$ & $-1,09$ & - & 0,82 & 2,03 & $-1,18$ & 576,1 & 0,71 & \\
\hline \multicolumn{2}{|c|}{ Melhor ajuste } & +1 & $-0,46$ & $-0,1$ & 0,8973 & - & - & - & - & - & - & - \\
\hline
\end{tabular}


Com a utilização do "solver" (aplicando-se o método Simplex) e através de uma planilha contendo um sistema lógico que tornou possivel imputar todas as restrições, variáveis de decisão e função objetivo (Figuras 9 e 10), pôde-se obter os resultados contendo as melhores respostas alcançadas através da otimização. Para isso foram utilizados os modelos estatísticos obtidos anteriormente através de regressão linear múltipla, conforme Equações 7, 8 e 9.

É possivel observar que ao se ajustarem os fatores nas configurações mostradas na Tabela 11, onde: $\mathrm{D}=-1$ (correspondente ao diâmetro 2,00 mm), $\mathrm{A}=1, \mathrm{~B}=0,042$ e $\mathrm{C}=-1$, puderam-se obter variáveis resposta que atendem às respectivas especificações e o erro médio obtido foi de $4,28 \%$.

\subsubsection{Aplicação para o diâmetro de $6,50 \mathrm{~mm}$}

0 método Simplex também foi aplicado para o diâmetro de $6,50 \mathrm{~mm}$. Para a aplicação desse método foi necessária a definição de:

- Especificações: Para o diâmetro de 6,50 mm (Tabela 10); onde se busca para a variável resposta limite de resistência à tração valores entre $1770 \mathrm{MPa}$ a $1980 \mathrm{MPa}$, para estricção, valores entre $40 \%$ a 56\% e, para dureza, valores entre $500 \mathrm{HB}$ a $560 \mathrm{HB}$. As especificações foram inseridas no "solver" conforme procedimento executado anteriormente.

- Variáveis de decisão: Nesse caso, as variáveis de decisão são as mesmas utilizadas anteriormente para o diâmetro de $2,00 \mathrm{~mm}$ e a sua inserção no "solver" segue o mesmo procedimento realizado anteriormente.

- Função objetivo: Nesse caso, a função objetivo foi a mesma executada para o outro diâmetro estudado e o procedimento de inserção no "solver" seguiu o mesmo procedimento detalhado anteriormente.

Tabela 13. Ajustes alcançados para fatores, utilizando os métodos de otimização (diâmetro 2,00 mm).

\begin{tabular}{ccc}
\hline Fatores & Ajuste (Desirability) & Ajuste (Simplex) \\
\hline A & -1 & 1 \\
B & $-0,0909$ & 0,0420 \\
C & 1 & -1 \\
\hline
\end{tabular}

Utilizando-se as Equações 7, 8 e 9 foi possível obter as melhores respostas para as propriedades mecânicas referentes ao diâmetro de $6,50 \mathrm{~mm}$, que são mostradas na Tabela 12.

É possivel observar que ao se ajustarem os fatores nas configurações mostradas na Tabela 12, onde: $\mathrm{D}=+1$ (correspondente ao diâmetro de $6,50 \mathrm{~mm}$ ), $\mathrm{A}=-0,46 ; \mathrm{B}=-0,1$ e $\mathrm{C}=0,8973$, pôde-se obter variáveis resposta que atendem às respectivas especificações e o erro médio obtido foi de 0,03\%.

\subsection{Análise comparativa entre os métodos de otimização}

Na Tabela 13 são mostrados os melhores ajustes dos fatores alcançados com a aplicação dos métodos Desirability e Simplex em relação ao diâmetro 2,00 $\mathrm{mm}$. É possivel observar que as configurações dos melhores ajustes para os métodos diferem

Porém, analisando os dados da Tabela 14, constatou-se que, para o diâmetro de 2,00 mm, as diferenças relativas à otimização entre os métodos aplicados não foi significativa, pois todos os métodos tiveram erros muito próximos, entre 4,28\% e 5,77\%, com variação considerada pequena. Também é importante ressaltar que essa medição do erro entre os valores preditos e valores "alvos" foi somente para verificar o quanto seria possível otimizar os ajustes dos fatores, para que se aproximassem das especificações nominais (alvo) requeridas, pois no dia a dia da fabricação os "alvos" poderão ser buscados de acordo com a necessidade da empresa.

$\mathrm{Na}$ Tabela 15, em relação ao diâmetro de $6,50 \mathrm{~mm}$, são mostrados os melhores ajustes dos fatores alcançados com a utilização dos métodos de otimização. É possível observar que as melhores configurações para o método Simplex são diferentes das melhores configurações alcançadas pelo método Desirability.

Porém, analisando-se os dados da Tabela 16, constatou-se que as diferenças relativas à otimização entre os métodos aplicados também não foram significativas para o diâmetro de $6,50 \mathrm{~mm}$, pois, ambos os métodos obtiveram erros muito próximos

Tabela 14. Resultados alcançados utilizando-se os métodos de otimização (diâmetro 2,00mm).

\begin{tabular}{|c|c|c|c|c|c|c|}
\hline & \multicolumn{3}{|c|}{ Respostas (Desirability) } & \multicolumn{3}{|c|}{ Respostas (Simplex) } \\
\hline & Tração (MPa) & Estricção (\%) & Dureza (HB) & Tração (MPa) & Estricção (\%) & Dureza (HB) \\
\hline Predição (modelo) & 2029,50 & 54,82 & 572,86 & 2040 & 53,50 & 576 \\
\hline Especificação mínima & 1930 & 40 & 545 & 1930 & 40 & 545 \\
\hline Especificação máxima & 2150 & 55 & 600 & 2150 & 55 & 600 \\
\hline Especificação (alvo) & 2040 & 47 & 572 & 2040 & 47 & 572 \\
\hline Erro médio & & 5,77 & & & 4,28 & \\
\hline
\end{tabular}


Tabela 15. Ajustes alcançados para fatores, utilizando os métodos de otimização (diâmetro 6,50 mm)

\begin{tabular}{ccc}
\hline Fatores & Ajuste (Desirability) & Ajuste (Simplex) \\
\hline A & -1 & $-0,4600$ \\
B & $-0,1919$ & $-0,1000$ \\
C & 1 & 0,8973 \\
\hline
\end{tabular}

Tabela 16. Resultados alcançados utilizando-se os métodos de otimização (diâmetro 6,50 mm).

\begin{tabular}{|c|c|c|c|c|c|c|}
\hline & \multicolumn{3}{|c|}{ Respostas (Desirability) } & \multicolumn{3}{|c|}{ Respostas (Simplex) } \\
\hline & Tração (MPa) & Estricção (\%) & Dureza (HB) & Tração (MPa) & Estricção (\%) & Dureza (HB) \\
\hline Predição (por modelo estatístico) & 1875,60 & 50,77 & 529,80 & 1875,20 & 48 & 529,60 \\
\hline Especificação mínima & 1770 & 40 & 500 & 1770 & 40 & 500 \\
\hline Especificação máxima & 1980 & 56 & 560 & 1980 & 56 & 560 \\
\hline Especificação (alvo) & 1875 & 48 & 530 & 1875 & 48 & 530 \\
\hline Erro médio (para cada método) & & & 1,95 & & & 0,03 \\
\hline
\end{tabular}

em relação à especificação nominal, variando entre 0,03\% e 1,95\%, com variação considerada pequena.

\section{Conclusões}

A metodologia de planejamento de experimentos com análise em blocos aplicada no processo de tratamento térmico de têmpera e revenimento em arames de aço SAE 9254 trefilado, com diâmetros de $2,00 \mathrm{~mm}$ e de $6,50 \mathrm{~mm}$ proporcionou a compreensão ampla da influência dos fatores nas propriedades mecânicas limite de resistência à tração, estricção e dureza.

Os resultados revelaram, através do teste de significância, que os fatores diâmetro, velocidade, temperatura de revenimento e concentração do polímero têm influência significativa nas propriedades mecânicas estudadas. Contudo, a metodologia regressão linear múltipla se mostrou eficiente para modelar estatisticamente o processo e a análise gráfica gerada pela aplicação do método Desirability possibilitou a visualização do comportamento das múltiplas respostas, simultaneamente, e a otimização do processo na melhor condição de ajustes dos fatores de entrada, proporcionando a melhor eficiência das variáveis de saída em relação às especificações. Além disso, com a utilização de mais um método de otimização, no caso o método Simplex (por programação linear), pôde-se comprovar que ambos os métodos alcançaram resultados muito similares na otimização das múltiplas respostas mecânicas em relação aos erros entre os valores preditos e as especificações requeridas.

Por meio das descobertas geradas por essa pesquisa busca-se ajustar de forma planejada o setup do forno de têmpera em ambiente produtivo, obtendo-se, assim, a redução da quantidade de ensaios laboratoriais iniciais e do tempo de espera desses resultados, cujo custo tem impacto direto nos indicadores financeiros da empresa. Tais custos têm relação com gastos em insumos para ensaios destrutivos, disponibilidade reduzida de horas-máquina de trabalho devido aos tempos de parada (aguardando resultados mecânicos) e alocação de mão de obra destinada à preparação (setup) dos fornos de têmpera. Todavia, além de afetar diretamente a produtividade, também afeta a qualidade do produto, pois sem uma simulação adequada de resultados não é possível alcançar-se com frequência o ajuste nominal ideal das múltiplas respostas de forma simultânea. Esse ajuste planejado deverá ser realizado através das predições, utilizando-se o modelo estatístico que, em conjunto com a aplicação dos métodos Desirabilitye Simplex, poderá proporcionar a otimização das múltiplas respostas nas melhores condições e garantindo o atendimento das especificações.

\section{Referências}

Benyounis, K. Y., \& Olabi, A. G. (2008). Optimization of different welding processes using statistical and numerical: a reference guide. Science Direct, 39(6), 483496.

Callister Junior, W. D. (2002). Uma introdução a engenharia e a ciências dos materiais (5. ed., pp. 589). Rio de Janeiro: LTC.

Derringer, G., \& Suich, R. (1980). Simultaneous optimization of several response variables. Journal of Quality Technology, 12(4), 214-219.

Granato, D., Branco, G. F., \& Calado, V. M. A. (2011). Experimental design and application of response surface methodology for process modelling and optimization: a review. Food Research International, 1, 1-14.

Lima, V. B. S., Balestrassi, P. P., \& Paiva, A. P. (2011) Otimização do desempenho de amplificadores de radio frequência banda larga: uma abordagem experimental. Produção, 21(1), 118-131. http://dx.doi.org/10.1590/ S0103-65132011005000005 
Marins, F. A. S. (2011). Introdução à pesquisa operacional (pp. 11-25). São Paulo: Cultura Acadêmica.

Mayers, A. M., \& Chawla, K. K. (1982). Princípios de metalurgia mecânica (2. ed.). São Paulo: Blucher.

Montgomery, C. D. (2010). Design and analysis of experiments (7th ed.) New York: John Wiley \& Sons.

Montgomery, D. C., \& Runger, G. C. (2003). Estatística aplicada e probabilidade para engenheiros (2. ed., pp. 230-320). Rio de Janeiro: LTC.

Paiva, E. J. (2008). Otimização de manufatura com múltiplas respostas baseadas em índices de capacidade (Master's thesis). Universidade Federal de Itajubá, Itajubá.
Silva, H. A., \& Silva, M. B. (2008). Aplicação de um projeto de experimentos (DOE) na soldagem de tubos de zircaloy-4. Produção \& Engenharia, 1(1), 41-52.

Wang, J., \& Wan, W. (2009). Application of desirability function based on neural network for optimizing biohydrogen production process. International Journal of Hydrogen Energy, 34(3), 1253-1259. http://dx.doi. org/10.1016/j.jjhydene.2008.11.055

Wu, F. C. (2005). Optimization of correlated multiple quality characteristics using desirability function. Quality Engineering, 17(1), 119-126. http://dx.doi.org/10.1081/ QEN-200028725

\title{
Application of methodologies Desirability and Simplex for optimization of the mechanical properties intempered steel wires
}

\begin{abstract}
The objective of this research is to show the application of the desirability method and simplex method in aiming to optimize the process of quench hardening and tempering in SAE 9254 draw steel wires. The values were generated by applying the methodology design of experiments with the block analysis, and the results revealed that all the variables used in the study have a significant influence on obtaining the mechanical properties investigated. For the statistical modeling, the multiple linear regression method was applied to obtain models that adequately represent the case in question. The results of the response variables (tensile strength, yield point and hardness) were submitted to the desirability method and the simplex method, and the process was optimized by adjusting the variables of the input in relation to the given specifications.
\end{abstract}

\section{Keywords}

Design of experiments. Multiple linear regression. Function desirability. Simplex methodology. 